\title{
Parâmetros de transferência de massa da extração do óleo de sementes de mangaba (Hancornia speciosa Gomes) e de graviola (Annona muricata L.) utilizando dióxido de carbono supercrítico
}

Mass transfer parameters of the extraction of mangaba (Hancornia speciosa Gomes) and graviola (Annona muricata L.) seeds using supercritical carbon dioxide

\author{
M. F. Santos ${ }^{1 *} ;$ J. J. Gomes ${ }^{1} ;$ D. Santos ${ }^{1} ;$ L. Cardozo-Filho²; E. de Jesus ${ }^{1}$ \\ ${ }^{1}$ Departamento de Engenharia Química, Programa de Pós-Graduação em Engenharia Química- PEQ, Universidade \\ Federal de Sergipe, 49100-000, São Cristovão-Sergipe, Brasil \\ ${ }^{2}$ Departamento de Engenharia Química, Universidade Estadual de Maringá, 87020-900, Maringá-Paraná, Brasil
}

*michel.chemie@gmail.com

(Recebido em 24 de julho de 2017; aceito em 18 de maio de 2018)

\begin{abstract}
O presente trabalho teve como objetivo estudar os parâmetros de transferência de massa do processo de extração de óleo de sementes de graviola (Annona muricata L.) e de mangaba (Hancornia speciosa Gomes) com $\mathrm{CO}_{2}$ supercrítico e avaliar o ajuste dos dados utilizando o modelo de Sovová. A condição de extração foi de $200 \mathrm{bar}, 40^{\circ} \mathrm{C}$, tempo de extração de $145 \mathrm{~min}$ e vazão de solvente de $2,0 \mathrm{~mL} / \mathrm{min}$. Os resultados mostraram que a extração não atingiu a etapa difusional. O coeficiente de transferência de massa foi de 0,0646 e $0,1381 \mathrm{~min}^{-1}$ para a mangaba e graviola, respectivamente. Os rendimentos obtidos foram aproximadamente $4,3 \%$ para a semente de mangaba e $7,0 \%$ para a semente de graviola.

Palavras-chave: óleo de sementes, extração com fluido supercrítico, parâmetros cinéticos
\end{abstract}

The objective of this work was to study the mass transfer parameters of the oil extraction process of graviola (Annona muricata L.) and mangaba (Hancornia speciosa Gomes) seeds with supercritical $\mathrm{CO}_{2}$ and to evaluate the fit of the data using the model of Sovová. The condition of extraction was $200 \mathrm{bar}, 40^{\circ} \mathrm{C}$, extraction time of $145 \mathrm{~min}$ and solvent flow of $2.0 \mathrm{~mL} / \mathrm{min}$. The data show that under these conditions was not enough to reach the diffusional stage. The mass transfer coefficients that were 0.0646 and $0.1381 \mathrm{~min}^{-1}$ for the mangaba and graviola, respectively. The yield obtained was $4.3 \%$ for the mangaba seed and $7.0 \%$ for the graviola seed.

Keywords: seed oil, supercritical fluid extraction, kinetic parameters

\section{INTRODUÇÃO}

O Brasil possui uma elevada biodiversidade e é um dos maiores produtores de frutas no mundo. As frutas são destinadas a alimentação humana na forma in natura ou são processadas e utilizadas pelas indústrias de cosméticos, farmacêuticas e alimentícias. No decorrer da cadeia produtiva essas indústrias geram resíduos agroindustriais, os quais muitas vezes não são destinados corretamente e isso reflete em impactos ambientais severos. Esses resíduos, em grande parte, são compostos de cascas e sementes e estudos são necessários para o seu aproveitamento para agregar valor aos produtos e gerar interesse econômico [1,2].

As sementes são matérias-primas comumente usadas para produção de óleo, além de possuírem componentes nutricionais expressivos, no entanto, poucos são os trabalhos voltados para resíduos do processamento da Graviola (Annona muricata L.) e Mangaba (Hancornia speciosa Gomes), as quais são frutas de grande consumo no Brasil, sendo a Mangaba uma fruta nativa do país $[3,4,5]$.

Os processos comumente utilizados na indústria extrativa de óleo de sementes usam solventes orgânicos como agentes de extração, porém exigem a adoção de operações unitárias de separação do óleo/solvente. Como alternativas outras tecnologias estão sendo avaliadas, a exemplo da extração com fluidos supercríticos (EFS), em que o solvente é mantido em condições de temperatura e pressão acima do seu estado crítico e tem como característica maior poder de solvatação do óleo no solvente, além de ser um processo em que o solvente é facilmente separado 
do óleo por simples despressurização do sistema, sendo considerado um processo verde, por não ser agressivo ao meio ambiente e a saúde humana. [6]

$\mathrm{O}$ dióxido de carbono $\left(\mathrm{CO}_{2}\right)$ é o solvente mais utilizado em processos de extração supercrítica por ser um solvente não tóxico, ideal para extração de óleo de matrizes naturais, ambientalmente amigável, possui alta difusividade e condições críticas de temperatura $\left(31,1^{\circ} \mathrm{C}\right)$ e pressão $(73,8 \mathrm{bar})$ relativamente baixas. Além disso, pode ser encontrado em condições de alta pureza e é um solvente de baixo custo, o que torna um solvente atrativo do ponto de vista econômico sendo considerado ideal para EFS $[7,8]$.

Diante deste contexto, o presente trabalho teve como objetivo estudar os parâmetros de transferência de massa do processo de extração de óleo de sementes de graviola (Annona muricata L.) e de mangaba (Hancornia speciosa Gomes) com $\mathrm{CO}_{2}$ supercrítico e avaliar o ajuste dos dados utilizando o modelo de Sovová.

\section{MATERIAL E MÉTODOS}

\subsection{Materiais}

As sementes de Mangaba e Graviola foram obtidas dos resíduos da produção de polpas das respectivas frutas. As amostras foram cedidas pela indústria de polpas Pomar do Brasil, localizada no município de Aracaju-Sergipe, Brasil. Para extração do óleo utilizou-se dióxido de carbono com 99,9\% de pureza proveniente da White Martins S.A. (Brasil).

\subsection{Preparo da matéria-prima}

As sementes foram previamente lavadas com água potável para retirada de resíduos de polpa e armazenadas a $-18^{\circ} \mathrm{C}$ em freezer convencional. Logo após, foram submetidas à secagem por liofilização e em seguida armazenadas em embalagens plásticas a vácuo. As amostras secas foram trituradas em moinho de facas (Solab, modelo: SL-30-Brasil).

\subsection{Extração por fluido supercrítico}

Os óleos das sementes foram obtidos por meio de extração supercrítica com o solvente $\mathrm{CO}_{2} \mathrm{em}$ seu estado supercrítico.

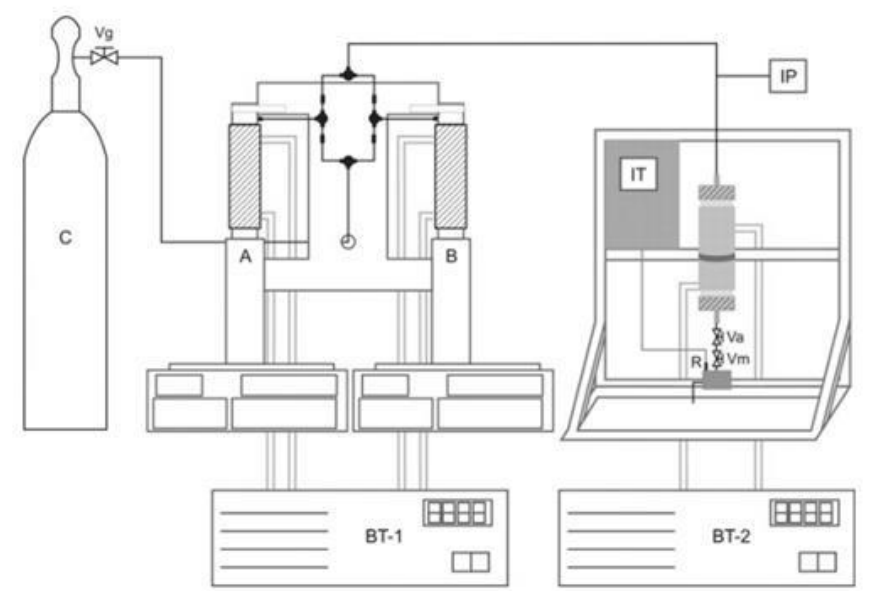

Figura 1: Aparato experimental utilizado para a extração com $\mathrm{CO}_{2}$ supercrítico. $\mathrm{C}$ : Cilindro de $\mathrm{CO}_{2}$; $\mathrm{Vg}$ : Válvula globo que permite a passagem de $\mathrm{CO}_{2}$ do cilindro até a bomba; A e B: Bomba do tipo seringa; BT1 e BT2: Banhos termostatizados; IP: Indicador de pressão; IT: Indicador de temperatura; Va: Válvula agulha para saída dos solventes saturados de óleo; Vm: Válvula micrométrica que permite regulagem do fluxo de solventes; R1: Reservatório de coleta do óleo [9].

Os experimentos foram realizados em escala de bancada, que consiste basicamente de um cilindro contendo o fluido pressurizado, dois banhos termostatizados, duas bombas seringas (ISCO, Modelo 500D) e um extrator com volume interno de 57,93 cm $\mathrm{cm}^{3}$ conforme é detalhado na Figura 1. 
Os experimentos foram realizados com $\mathrm{CO}_{2}$ supercrítico em 200 bar, $40^{\circ} \mathrm{C}$, vazão de solvente de $2 \mathrm{~mL} / \mathrm{min}$ e tempo de extração de 145 minutos. Inicialmente o solvente era bombeado e ficava em contato com a amostra por um tempo de 30 minutos até estabilização do sistema. O óleo era coletado em frasco âmbar a partir da abertura de duas válvulas uma macrométrica e uma micrométrica. Para obtenção da cinética de extração o frasco contendo a massa de óleo extraída era pesada e reconectada ao equipamento de 5 em 5 minutos e após 25 minutos de 15 em 15 minutos. O rendimento de extração foi calculado a partir da razão entre a massa de óleo extraído pela massa de semente utilizada para extração.

2.4 Estimação dos parâmetros de transferência de massa da extração do óleo

Os parâmetros de transferência foram estimados por meio do software STATISTICA versão 10 da Statsoft usando o modelo proposto por Sovová [10] descrito pelas Equações de (1) a (7).

Fase 1: $\mathrm{t}<\mathrm{t}_{\mathrm{CER}}$

$$
m_{\text {oleo }}=\dot{m}_{F} Y_{S} t[1-\exp (-Z)]
$$

Fase 2: $\mathrm{t}_{\mathrm{CER}} \leq \mathrm{t} \leq \mathrm{t}_{\mathrm{FER}}$

$$
m_{\text {oleo }}=\dot{m}_{F} Y_{S}\left[t-t_{C E R} \exp \left(\frac{Z Y_{S}}{W X_{0}} \ln \left\{\frac{1}{1-r}\left(\exp \left(\frac{W m_{F}}{m_{S}}\right)\left(t_{C E R}-t\right)-r\right)\right\}-Z\right)\right]
$$

Fase 3: $\mathrm{t}>\mathrm{t}_{\mathrm{FER}}$

$$
m_{\text {oleo }}=m_{S}\left[X_{0}-\frac{Y_{S}}{W} \ln \left\{1+\left(\exp \left(\frac{W X_{0}}{Y_{S}}\right)-1\right) \exp \left(\frac{W m_{F}}{m_{S}}\right)\left(t_{C E R}-t\right) r\right\}\right]
$$

Sendo:

$$
\begin{aligned}
& Z=\frac{k_{F} a m_{S} \rho_{F}}{\mathrm{~m}_{F} \rho_{S}} \\
& W=\frac{m_{S} k_{S} a}{m_{F}(1-\varepsilon)} \\
& t_{C E R}=\frac{(1-r) m_{S} X_{0}}{Y_{S} Z m_{F}} \\
& t_{F E R}=t_{C E R}+\frac{m_{S}}{W m_{F}} \ln \left[r+(1-r) \exp \left(\frac{W X_{0}}{Y_{S}}\right)\right]
\end{aligned}
$$

Onde: $t_{C E R}$ é o tempo de término do primeiro período de extração (min), $m_{\text {óleo }}$ é a massa de óleo $(\mathrm{g}), Y_{S}$ é a solubilidade de óleo no solvente (g oleo/g solvente), $\dot{\mathrm{m}}_{F}$ é a vazão mássica do solvente (g/min), $t$ é o tempo de extração (min), $Z$ e $W$ são parâmetros adimensionais do modelo, $X_{0}$ é a concentração inicial do óleo na matriz sólida ( $\mathrm{g}_{\text {óleo }} / \mathrm{g}_{\text {sólido }}$ ), $r$ é a fração de óleo facilmente 
acessível $\left(\mathrm{X}_{\mathrm{P}} / \mathrm{X}_{0}\right), m_{S}$ é a massa de sólido isenta de óleo $(\mathrm{g}), k_{F} a$ é o coeficiente de transferência de massa no filme externo $\left(\mathrm{min}^{-1}\right), \rho_{F}$ é a densidade do solvente $\left(\mathrm{g} / \mathrm{cm}^{3}\right), \rho_{S}$ é a densidade do sólido $\left(\mathrm{g} / \mathrm{cm}^{3}\right), k_{S} a$ coeficiente de transferência de massa no sólido $\left(\mathrm{min}^{-1}\right), \varepsilon$ é a porosidade, $t_{F E R}$ é o tempo de término do segundo período de extração (min) e r representa a fração de óleo de fácil acesso.

A soma dos erros ao quadrado foi utilizada como função objetivo $(F)$ conforme Equação (8).

$$
F=\sum_{j=1}^{N}\left(m_{\text {oleo }_{j}}^{\text {Calc }_{\text {ol }}}-m_{\text {oleo }_{j}}^{\text {Exp }}\right)^{2}
$$

Sendo $m_{\text {oleo }_{j}}^{\text {Calc }}$ a massa de óleo extraída obtida pelo modelo de Sovová; $m_{\text {oleo }}^{\text {Exp é a massa de óleo }}$ obtida experimentalmente e $N$ o número de pontos experimentais.

O coeficiente de determinação $\left(\mathrm{r}^{2}\right)$ para o modelo foi calculado de acordo com a Equação (9).

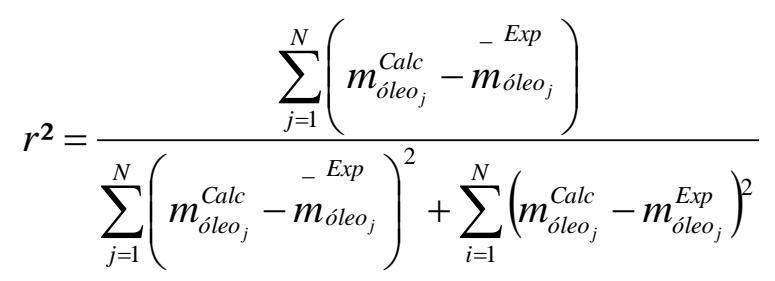

$$
\begin{aligned}
& \text { - Exp }
\end{aligned}
$$

Em que $m_{\text {óleo }}$ é a média dos valores experimentais.

\section{RESULTADOS E DISCUSSÃO}

A Figura 2 mostra a cinética de extração para as sementes de mangaba e graviola. O rendimento obtido para as duas sementes foi de $43,0 \mathrm{mg} / \mathrm{g}(4,29 \%)$ e $70 \mathrm{mg} / \mathrm{g}(6,97 \%)$ de semente para a mangaba e graviola, respectivamente. Esses dados mostram-se superiores aos dados encontrados por [11] na extração de óleo de sementes de uva com $\mathrm{CO}_{2}$ supercrítico, com $8,5 \mathrm{mg} / \mathrm{g}$ de semente, na condição de 200 bar e $40^{\circ} \mathrm{C}$.

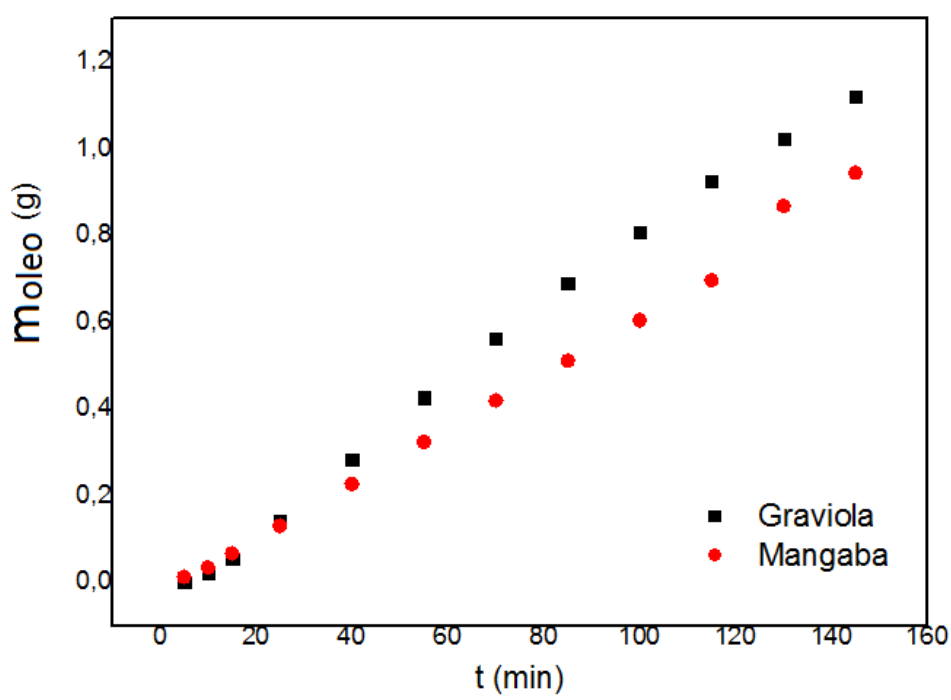

Figura 2: Cinética de extração

O modelo Sovová inclui 3 fases de extração em que cada estágio é controlado por fenômenos de transferência de massa. A primeira fase também conhecida como CER é uma fase em que o óleo de fácil acesso, proveniente da quebra de células na moagem, é facilmente extraído e a taxa de 
transferência de massa é constante. O segundo estágio é um período de decrescimento da taxa de extração (FER). E o último estágio que é controlado pela difusão (DCR) [12]. É possível observar na Figura 2, que para as duas cinéticas não foram obtidas as curvas das etapas FER e DCR, sendo o tempo de extração uma variável de importância no processo para atingir a etapa difusional, este comportamento em processos de EFS com dióxido de carbono também foi observado em [13], onde não foi alcançada a etapa difusional. Este comportamento também pode estar relacionado à disponibilidade de óleo na superfície das sementes trituradas.

A Tabela 1 mostra os dados da condição experimental utilizada na extração e os dados de entrada para obtenção dos parâmetros da modelagem do estágio CER da curva de extração das duas sementes. A solubilidade foi obtida através do método dinâmico equivalente ao coeficiente angular da parte linear da curva representada na Figura 2.

\begin{tabular}{lll}
\multicolumn{2}{c}{ Tabela 1: Condições experimentais e parâmetros da extração com fluido supercrítico } \\
\cline { 2 - 3 } Parâmetros & Mangaba & Semente \\
\hline Temperatura $\left({ }^{\circ} \mathrm{C}\right)$ & 40 & 40 \\
Pressão $($ bar $)$ & 200 & 200 \\
Densidade do $\mathrm{CO}_{2}\left(\mathrm{~g} / \mathrm{cm}^{3}\right)$ & 0,841 & 0,841 \\
$\mathrm{X}_{0}\left(\mathrm{~g}_{\text {óleo }} / \mathrm{g}_{\text {sólido }}\right)$ & 0,043 & 0,07 \\
Vazão de $\mathrm{CO}_{2}(\mathrm{~mL} / \mathrm{min})$ & 2 & 2 \\
Massa de sementes-alimentação $(\mathrm{g})$ & 22 & 16 \\
Diâmetro da partícula $(\mathrm{mm})$ & 2,10 & 1,58 \\
Porosidade & 0,66 & 0,77 \\
Densidade do sólido $\left(\mathrm{g} / \mathrm{cm}^{3}\right)$ & 1,14 & 1,24 \\
Vazão mássica de $\mathrm{CO}(\mathrm{g} / \mathrm{min})$ & 1,682 & 1,682 \\
Massa de sementes após extração $(\mathrm{g})$ & 21,06 & 14,88 \\
Solubilidade $\left(\mathrm{g}_{\text {óleo }} / \mathrm{g}_{\text {solvente }}\right)$ & 0,0066 & 0,0083 \\
\hline
\end{tabular}

A abordagem deste estudo está particularmente interessada em descrever a etapa CER conforme foi obtida nos experimentos e exposta na Figura 2. Os parâmetros ajustados do modelo Sovová são apresentados na Tabela 2 . O coeficiente de transferência de massa no filme externo $\left(\mathrm{k}_{\mathrm{F}} \mathrm{a},\right)$ foi obtido através da Equação (4).

Tabela 2: Parâmetros do modelo Sovová para as extrações de óleo com $\mathrm{CO}_{2}$ supercrítico

\begin{tabular}{lll}
\hline & \multicolumn{2}{c}{ Parâmetros } \\
Semente & $\mathrm{Z}$ & $\mathrm{k}_{\mathrm{F}}\left(\min ^{-1}\right)$ \\
Mangaba & 0,5969 & 0,0646 \\
Graviola & 0,8287 & 0,1381 \\
\hline
\end{tabular}

O coeficiente de extração na fase fluida indica maior valor para semente de graviola que reflete em um maior rendimento. Isso mostra que esse parâmetro está relacionado com a extração de óleo de fácil acesso.

As curvas cinéticas com o ajuste pelo modelo de Sovová são apresentadas na Figura 3, onde os coeficientes de determinação $\left(\mathrm{r}^{2}\right)$ foram maiores que 0,99 para os processos de extração das sementes. 


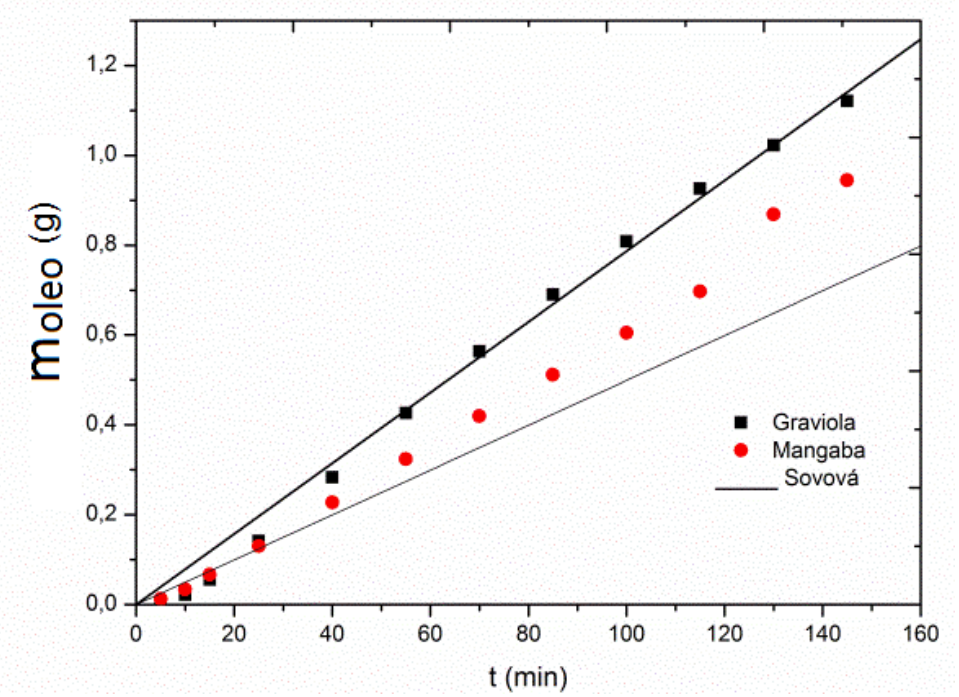

Figura 3: Curvas de cinética de extração e ajuste pelo modelo Sovová

\section{CONCLUSÃO}

Os resultados mostraram que o modelo de Sovová pode ser utilizado para estudar os parâmetros de transferência de massa da extração do óleo de sementes de mangaba e de graviola. A extração do óleo de sementes de graviola mostrou-se mais favorável, decorrente provavelmente de uma maior disponibilidade deste na matriz, sendo esta conclusão comprovada pelo maior valor do coeficiente de massa na fase fluídica $\left(\mathrm{k}_{\mathrm{F}} \mathrm{a}\right)$ obtido quando da extração do óleo de graviola.

\section{AGRADECIMENTOS}

Os pesquisadores agradecem a Universidade Federal de Sergipe (UFS), Universidade Estadual de Maringá (UEM), CAPES, CNPq e a Fundação de apoio à pesquisa do Estado de Sergipe (FAPITEC).

\section{REFERÊNCIAS BIBLIOGRÁFICAS}

1. Abud AKDS, Narain N. Incorporação da farinha de resíduo do processamento de polpa de fruta em biscoitos: uma alternativa de combate ao desperdício. Brazilian J Food Technol. 2009;12(4):257-265, doi:10.4260/BJFT2009800900020.

2. Do Nascimento Filho WB, Franco CR. Avaliação do potencial dos resíduos produzidos através do processamento agroindustrial no Brasil. Rev Virtual Quim. 2015;7(6):1968-1987, doi:10.5935/19846835.20150116.

3. Cardoso LDM, Reis BDL, Oliveira DDS, Pinheiro-Sant'Ana HM. Mangaba (Hancornia speciosa Gomes) from the Brazilian Cerrado: Nutritional value, carotenoids and antioxidant vitamins. Fruits. 2014;69(2):89-99, doi:10.1051/fruits/2013105.

4. Kimbonguila A, Nzikou JM, Matos L, Loum B, Ndangui CB, Pambou-Tobi NPG. Proximate composition and physicochemical properties on the seeds and oil of Annona muricata grown in Congo-Brazzaville. Res J Environ Earth Sci. 2010;2(1):13-18.

5. Santos RM, Santos AO, Sussuchi EM, Nascimento JS, Lima AS, Freitas LS. Pyrolysis of mangaba seed: Production and characterization of bio-oil. Bioresour Technol. 2015;196:43-48, doi:10.1016/j.biortech.2015.07.060.

6. De Melo MMR, Silvestre AJD, Silva CM. Supercritical fluid extraction of vegetable matrices: Applications, trends and future perspectives of a convincing green technology. J Supercrit Fluids. 2014;92:115-176, doi:10.1016/j.supflu.2014.04.007.

7. Huang Z, Shi X-H, Jiang W-J. Theoretical models for supercritical fluid extraction. J Chromatogr A. 2012;1250:2-26, doi:10.1016/j.chroma.2012.04.032.

8. Marques LLM, Panizzon GP, Aguiar BAA, et al. Guaraná (Paullinia cupana) seeds: Selective 
supercritical extraction of phenolic compounds. Food Chem. 2016;212:703-711, doi:10.1016/j.foodchem.2016.06.028.

9. Garcia VADS, Cabral VF, Zanoelo ÉF, Da Silva C, Filho LC. Extraction of Mucuna seed oil using supercritical carbon dioxide to increase the concentration of 1-Dopa in the defatted meal. J Supercrit Fluids. 2012;69:75-81, doi:10.1016/j.supflu.2012.05.007.

10. Sovova $\mathrm{H}$. Rate of the vegetable oil extraction with supercritical $\mathrm{CO}_{2}$ - modelling of extraction curves. Chem Eng Sci. 1994;49(3):409-414, doi:10.1016/0009-2509(94)87012-8.

11. Pérez C, Ruiz del Castillo ML, Gil C, Blanch GP, Flores G. Supercritical fluid extraction of grape seeds: extract chemical composition, antioxidant activity and inhibition of nitrite production in LPS-stimulated Raw 264.7 cells. Food Funct. 2015;6(8):2607-2613, doi:10.1039/C5FO00325C.

12. Leitão NCMCS, Prado GHC, Veggi PC, Meireles MAA, Pereira CG. Anacardium occidentale L. leaves extraction via SFE: Global yields, extraction kinetics, mathematical modeling and economic evaluation. J Supercrit Fluids. 2013;78:114-123, doi:10.1016/j.supflu.2013.03.024.

13. Czaikoski K, Mesomo MC, Kruger RL, Queiroga CL, Corazza ML.Extraction of campomanesia xantocarpa fruit using supercritical $\mathrm{CO}_{2}$ and bioactivity assessments. J Supercrit Fluids. 2015;98:79-85, doi:10.1016/j.supflu.2015.01.006. 